Document downloaded from:

http://hdl.handle.net/10251/52542

This paper must be cited as:

Cordero Barbero, A.; Torregrosa Sánchez, JR. (2011). A class of Steffensen type methods with optimal order of convergente. Applied Mathematics and Computation. 217(19):76537659. doi:10.1016/j.amc.2011.02.067.

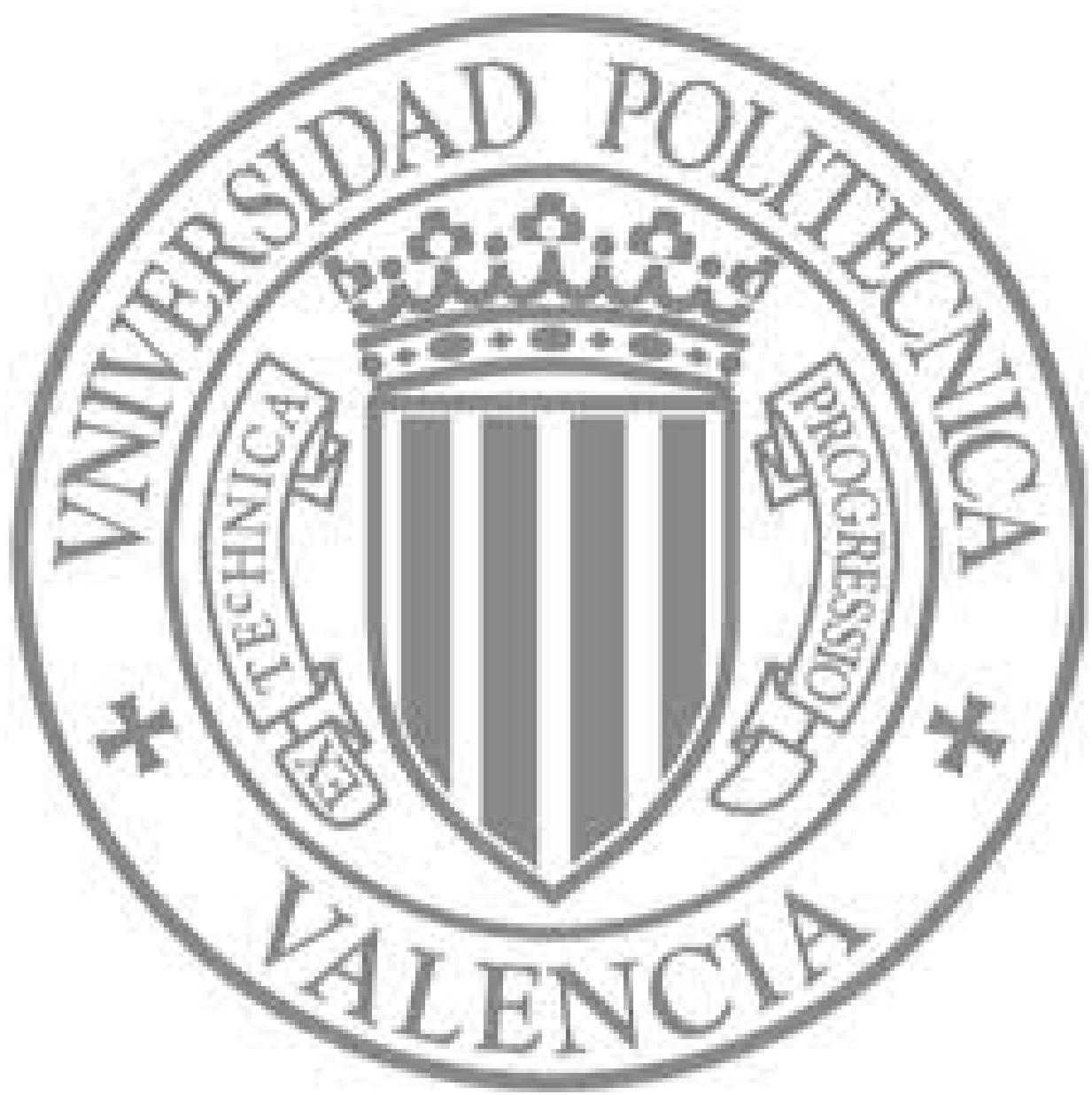

The final publication is available at

http://dx.doi.org/10.1016/j.amc.2011.02.067

Copyright Elsevier 


\title{
A class of Steffensen type methods with optimal order of convergence $\star$
}

\author{
Alicia Cordero, Juan R. Torregrosa * \\ Instituto de Matemática Multidisciplinar, \\ Universidad Politécnica de Valencia, \\ Camino de Vera, s/n, 46022 \\ Valencia, Spain
}

\begin{abstract}
In this paper, a family of Steffensen type methods of fourth-order convergence for solving nonlinear smooth equations is suggested. In the proposed methods, a linear combination of divided diferences is used to get a better approximation to the derivative of the given function. Each derivative-free member of the family requires only three evaluations of the given function per iteration. Therefore, this class of methods has efficiency index equal to 1.587. Kung and Traub conjectured that the order of convergence of any multipoint method without memory cannot exceed the bound $2^{d-1}$, where $d$ is the number of functional evaluations per step. The new class of methods agrees with this conjecture for the case $d=3$. Numerical examples are made to show the performance of the presented methods, on smooth and nonsmooth equations, and to compare with other ones.
\end{abstract}

Key words: Nonlinear equations, Iterative methods, Convergence order, Efficiency index, Steffensen's method, Derivative free method

\section{Introduction}

Solving nonlinear equations is a classical problem which has interesting applications in various branches of science and engineering. In this study, we

ऋ This research was supported by Ministerio de Ciencia y Tecnología MTM201018539

* Correspondig author

Email addresses: acordero@mat.upv.es (Alicia Cordero), jrtorre@mat.upv.es (Juan R. Torregrosa ). 
describe new iterative methods to find a simple root $x^{*}$ of a nonlinear equation $f(x)=0$, where $f: I \subset \mathbb{R} \rightarrow \mathbb{R}$ is a scalar function on an open interval $I$. The known Newton's method for finding $x^{*}$ uses the iterative expression

$$
x_{k+1}=x_{k}-\frac{f\left(x_{k}\right)}{f^{\prime}\left(x_{k}\right)}, \quad k=0,1, \ldots
$$

which converges quadratically in some neighborhood of $x^{*}$. If the derivative $f^{\prime}\left(x_{k}\right)$ is replaced by the forward-difference approximation

$$
f^{\prime}\left(x_{k}\right) \approx \frac{f\left(x_{k}+f\left(x_{k}\right)\right)-f\left(x_{k}\right)}{f\left(x_{k}\right)}
$$

the Newton's method becomes

$$
x_{k+1}=x_{k}-\frac{\left(f\left(x_{k}\right)\right)^{2}}{f\left(x_{k}+f\left(x_{k}\right)\right)-f\left(x_{k}\right)},
$$

which is the known Steffensen's method (SM), (see [1]). This scheme is a tough competitor of Newton's method. Both methods are of second order, both require two functional evaluations per step, but in contrast to Newton's method, Steffensen's method is derivative-free.

To improve the convergence properties, many variants of Steffensen's method has been proposed in the last years. Some of these methods use forward or central divided differences for approximating the derivatives. For example, Jain in [2] proposed a Steffensen-secant method (SSM) deformed from Newtonsecant as follows:

$$
x_{k+1}=x_{k}-\frac{\left(f\left(x_{k}\right)\right)^{3}}{\left[f\left(x_{k}+f\left(x_{k}\right)\right)-f\left(x_{k}\right)\right]\left[f\left(x_{k}\right)-f\left(y_{k}\right)\right]},
$$

where $y_{k}$ is the kth iteration of the Steffensen's method. This method only uses three functional evaluations per step and arrives third-order convergence.

Dehghan and Hajarian [3] proposed a variant of Steffensen's method (DHM), which is written as

$$
x_{k+1}=x_{k}-\frac{2 f\left(x_{k}\right)\left[f\left(z_{k+1}\right)-f\left(x_{k}\right)\right]}{f\left(x_{k}+f\left(x_{k}\right)\right)-f\left(x_{k}-f\left(x_{k}\right)\right)},
$$

where $z_{k+1}=x_{k}-\frac{2\left(f\left(x_{k}\right)\right)^{2}}{f\left(x_{k}+f\left(x_{k}\right)\right)-f\left(x_{k}-f\left(x_{k}\right)\right)}$. The method is obtained by replacing the forward-difference approximation in Steffensen's method by the centraldifference approximation. However, it is still a method of third order and requires four functional evaluations per iteration.

Recently, Ren et al. derive in [4] a one-parameter class of fourth-order methods (RM) with three functional evaluations per step. In these methods, an 
interpolation polynomial of order three is used to get a better approximation to the derivative of the given function. The iterative expression is:

$$
x_{k+1}=y_{k}-\frac{f\left(y_{k}\right)}{f\left[x_{k}, y_{k}\right]+f\left[y_{k}, z_{k}\right]-f\left[x_{k}, z_{k}\right]+a\left(y_{k}-x_{k}\right)\left(y_{k}-z_{k}\right)} \text {, }
$$

where $y_{k}$ is the approximation of the Steffensen's method and $f[\cdot, \cdot]$ is the divided difference of order one. Other Steffensen type methods and their applications are also discussed by Zheng et al. in [5] and by Feng and He in [6].

To improve the local order of convergence, a known technique consist of the composition of two iterative methods of order $p$ and $q$, respectively, to obtain a method of order $p q$ (see [1]). As the order of an iterative method increases, so does the number of functional evaluations per step. The efficiency index (see [7]) gives a measure of the balance between those quantities, according to the formula $p^{1 / d}$, where $p$ is the order of convergence of the method and $d$ the number of functional evaluations per step. Kung and Traub conjectured in [8] that the order of convergence of any multipoint method cannot exceed the bound $2^{d-1}$, (called the optimal order). Thus, the optimal order for a method with 3 functional evaluations per step would be 4 . The methods (RM) are some of optimal fourth order methods.

In this paper, we derive a one-parameter family of fourth-order methods for solving nonlinear equations. In the proposed methods, we compose Steffensen and Newton's methods and we use a linear combination of two divided differences of order one to get a better approximation to the derivative of the given function. Each member of the family requires three evaluations of the function $f(x)$, therefore this class of methods has efficiency index $4^{1 / 3} \approx 1.587$, which is higher than $2^{1 / 2} \approx 1.414$ of Steffensen method, $3^{1 / 3} \approx 1.442$ of Jain's method $(1)$ and $3^{1 / 4} \approx 1.316$ of Dehghan and Hajarian's method (2). The new family of methods reaches the optimal order of convergence four, conjectured by Kung and Traub.

The rest of the paper is organized as follows: in Section 2 we describe our family of methods and we show the order of convergence of it. In Section 3, different numerical test confirm the theoretical results and allow us to compare this family with other known methods mentioned in this section.

\section{The methods and analysis of convergence}

It is easy to see that the following Steffensen-Newton scheme 


$$
\begin{aligned}
y_{k} & =x_{k}-\frac{\left(f\left(x_{k}\right)\right)^{2}}{f\left(z_{k}\right)-f\left(x_{k}\right)}, \\
x_{k+1} & =y_{k}-\frac{f\left(y_{k}\right)}{f^{\prime}\left(y_{k}\right)},
\end{aligned}
$$

where $z_{k}=x_{k}+f\left(x_{k}\right)$, is of fourth-order. In order to avoid the evaluation of the first derivative, we consider different approximations of it. Firstly, we replace $f^{\prime}\left(y_{k}\right)$ by the divided difference of order one

$$
f^{\prime}\left(y_{k}\right) \approx \frac{a f\left(y_{k}\right)-b f\left(z_{k}\right)}{y_{k}-z_{k}}
$$

where $a, b \in \mathbb{R}$ are parameters. It can be shown that for any values of $a$ and $b$ the described method has an order of convergence less than or equal to three. So, in order to preserve the order of convergence of (4) we replace $f^{\prime}\left(y_{k}\right)$ by a linear combination of two divided difference

$$
f^{\prime}\left(y_{k}\right) \approx \frac{a f\left(y_{k}\right)-b f\left(z_{k}\right)}{y_{k}-z_{k}}+\frac{c f\left(y_{k}\right)-d f\left(x_{k}\right)}{y_{k}-x_{k}},
$$

where $a, b, c, d \in \mathbb{R}$ are parameters. We are going to prove that for some values of the parameters the family of methods described by

$$
\begin{gathered}
y_{k}=x_{k}-\frac{\left(f\left(x_{k}\right)\right)^{2}}{f\left(z_{k}\right)-f\left(x_{k}\right)}, \\
x_{k+1}=y_{k}-\frac{f\left(y_{k}\right)}{\frac{a f\left(y_{k}\right)-b f\left(z_{k}\right)}{y_{k}-z_{k}}+\frac{c f\left(y_{k}\right)-d f\left(x_{k}\right)}{y_{k}-x_{k}}},
\end{gathered}
$$

is of fourth-order and we will denote it by $\mathrm{Op} 4$.

Theorem 1 Let $x^{*} \in I$ be a simple zero of a sufficiently differentiable function $f: I \subseteq \mathbb{R} \longrightarrow \mathbb{R}$ in an open interval I. If $x_{0}$ is sufficiently close to $x^{*}$, then the iterative method defined by (6) has optimal fourth convergence order for $a=c=1$ and $b+d=1$, and satisfies the error equation

$$
e_{k+1}=\left(1+f^{\prime}\left(x^{*}\right)\right)^{2} c_{2}\left(c_{2}^{2}-c_{3}\right) e_{k}^{4}+O\left(e_{k}^{5}\right),
$$

where $e_{k}=x_{k}-x^{*}$ and $c_{k}=\frac{1}{k !} \frac{f^{(k)}\left(x^{*}\right)}{f^{\prime}\left(x^{*}\right)}, k=2,3, \ldots$

Proof: By using Taylor's expansion around $x=x^{*}$, noting that $e_{k}=x_{k}-x^{*}$ and $f\left(x^{*}\right)=0$, we have

$$
f\left(x_{k}\right)=f^{\prime}\left(x^{*}\right)\left[e_{k}+c_{2} e_{k}^{2}+c_{3} e_{k}^{3}+c_{4} e_{k}^{4}\right]+O\left(e_{k}^{5}\right)
$$


and

$$
z_{k}-x^{*}=\left(1+f^{\prime}\left(x^{*}\right)\right) e_{k}+f^{\prime}\left(x^{*}\right) c_{2} e_{k}^{2}+f^{\prime}\left(x^{*}\right) c_{3} e_{k}^{3}+f^{\prime}\left(x^{*}\right) c_{4} e_{k}^{4}+O\left(e_{k}^{5}\right),
$$

then

$$
\begin{aligned}
f\left(z_{k}\right) & =f^{\prime}\left(x^{*}\right)\left(1+f^{\prime}\left(x^{*}\right)\right) e_{k}+f^{\prime}\left(x^{*}\right)\left(f^{\prime}\left(x^{*}\right)+\left(1+f^{\prime}\left(x^{*}\right)\right)^{2}\right) c_{2} e_{k}^{2}+ \\
& +f^{\prime}\left(x^{*}\right)\left[2 f^{\prime}\left(x^{*}\right)\left(1+f^{\prime}\left(x^{*}\right)\right) c_{2}^{2}+f^{\prime}\left(x^{*}\right) c_{3}+\left(1+f^{\prime}\left(x^{*}\right)\right)^{3} c_{3}\right] e_{k}^{3}+ \\
& +f^{\prime}\left(x^{*}\right)\left[f^{\prime}\left(x^{*}\right)^{2} c_{2}^{3}+f^{\prime}\left(x^{*}\right)\left(5+8 f^{\prime}\left(x^{*}\right)+3 f^{\prime}\left(x^{*}\right)^{2}\right) c_{2} c_{3}+\right. \\
& \left.+\left(1+5 f^{\prime}\left(x^{*}\right)+6 f^{\prime}\left(x^{*}\right)^{2}+4 f^{\prime}\left(x^{*}\right)^{3}+f^{\prime}\left(x^{*}\right)^{4}\right) c_{4}\right] e_{k}^{4}+O\left(e_{k}^{5}\right) .
\end{aligned}
$$

By substituting (7) and (9) in the expression of $y_{k}$ in (6), we obtain

$$
\begin{aligned}
y_{k}-x^{*} & =\left(1+f^{\prime}\left(x^{*}\right)\right) c_{2} e_{k}^{2}+\left[-\left(2+2 f^{\prime}\left(x^{*}\right)+f^{\prime}\left(x^{*}\right)^{2}\right) c_{2}^{2}+\left(2+3 f^{\prime}\left(x^{*}\right)+f^{\prime}\left(x^{*}\right)^{2}\right) c_{3}\right] e_{k}^{3}+ \\
& +\left[\left(4+5 f^{\prime}\left(x^{*}\right)+3 f^{\prime}\left(x^{*}\right)^{2}+f^{\prime}\left(x^{*}\right)^{3}\right) c_{2}^{3}-\left(7+10 f^{\prime}\left(x^{*}\right)+7 f^{\prime}\left(x^{*}\right)^{2}+2 f^{\prime}\left(x^{*}\right)^{3}\right) c_{2} c_{3}+\right. \\
& \left.+\left(3+6 f^{\prime}\left(x^{*}\right)+4 f^{\prime}\left(x^{*}\right)^{2}+f^{\prime}\left(x^{*}\right)^{3}\right) c_{4}\right] e_{k}^{4}+O\left(e_{k}^{5}\right)
\end{aligned}
$$

and using again Taylor's expansion

$$
\begin{aligned}
f\left(y_{k}\right) & =f^{\prime}\left(x^{*}\right)\left(1+f^{\prime}\left(x^{*}\right)\right) c_{2} e_{k}^{2}+ \\
& +f^{\prime}\left(x^{*}\right)\left[-\left(2+2 f^{\prime}\left(x^{*}\right)+f^{\prime}\left(x^{*}\right)^{2}\right) c_{2}^{2}+\left(2+3 f^{\prime}\left(x^{*}\right)+f^{\prime}\left(x^{*}\right)^{2}\right) c_{3}\right] e_{k}^{3}+ \\
& +f^{\prime}\left(x^{*}\right)\left[\left(5+7 f^{\prime}\left(x^{*}\right)+4 f^{\prime}\left(x^{*}\right)^{2}+f^{\prime}\left(x^{*}\right)^{3}\right) c_{2}^{3}-\right. \\
& -\left(7+10 f^{\prime}\left(x^{*}\right)+7 f^{\prime}\left(x^{*}\right)^{2}+2 f^{\prime}\left(x^{*}\right)^{3}\right) c_{2} c_{3}+ \\
& \left.+\left(3+6 f^{\prime}\left(x^{*}\right)+4 f^{\prime}\left(x^{*}\right)^{2}+f^{\prime}\left(x^{*}\right)^{3}\right) c_{4}\right] e_{k}^{4}+O\left(e_{k}^{5}\right) .
\end{aligned}
$$

Now, the quotient $Q=\frac{f\left(y_{k}\right)}{\frac{a f\left(y_{k}\right)-b f\left(z_{k}\right)}{y_{k}-z_{k}}+\frac{c f\left(y_{k}\right)-d f\left(x_{k}\right)}{y_{k}-x_{k}}}$ can be written as

$$
\begin{aligned}
Q & =(b+d) f^{\prime}\left(x^{*}\right)+f^{\prime}\left(x^{*}\right)\left(-a-c+2 d-c f^{\prime}\left(x^{*}\right)+d f^{\prime}\left(x^{*}\right)+b\left(2+f^{\prime}\left(x^{*}\right)\right)\right) c_{2} e_{k}+ \\
& +f^{\prime}\left(x^{*}\right)\left[\left(c+(b+d) f^{\prime}\left(x^{*}\right)+a\left(1+f^{\prime}\left(x^{*}\right)\right) c_{2}^{2}\right)+\right. \\
& +\left(-2 c+3 d-3 c f^{\prime}\left(x^{*}\right)+3 d f^{\prime}\left(x^{*}\right)-c f^{\prime}\left(x^{*}\right)^{2}+d f^{\prime}\left(x^{*}\right)^{2}-a\left(2+f^{\prime}\left(x^{*}\right)+\right.\right. \\
& \left.\left.\left.+b\left(3+3 f^{\prime}\left(x^{*}\right)+f^{\prime}\left(x^{*}\right)^{2}\right)\right) c_{3}\right)\right] e_{k}^{2}+O\left(e_{k}^{3}\right) .
\end{aligned}
$$

So,

$$
e_{k+1}=\frac{(-1+b+d)\left(1+f^{\prime}\left(x^{*}\right)\right) c_{2}}{b+d} e_{k}^{2}+O\left(e_{k}^{3}\right)
$$

In order to get order three, we need that $b+d=1$. Assuming this condition, the error expression becomes

$$
e_{k+1}=-\left(1+f^{\prime}\left(x^{*}\right)\right)\left(-2+a+c-f^{\prime}\left(x^{*}\right)+c f^{\prime}\left(x^{*}\right)\right) c_{2}^{2} e_{k}^{3}+O\left(e_{k}^{4}\right) .
$$


Now, we choose $c=1$ to remove the term $f^{\prime}\left(x^{*}\right)$ of the coefficient of $e_{k}^{3}$, and the expression of the error is

$$
e_{k+1}=-(1-a)\left(1+f^{\prime}\left(x^{*}\right)\right) c_{2}^{2} e_{k}^{3}+O\left(e_{k}^{4}\right)
$$

Then, taking $a=1$ we obtain a family of methods whose convergence order is four and its error expression is

$$
e_{k+1}=\left(1+f^{\prime}\left(x^{*}\right)\right)^{2} c_{2}\left(c_{2}^{2}-c_{3}\right) e_{k}^{4}+O\left(e_{k}^{5}\right)
$$

Let us remark that, in terms of computational cost, the developed methods require only three functional evaluations per step. So, they have efficiency indices $4^{1 / 3}=1.587$, that is, the new family of methods reaches the optimal order of convergence four, conjectured by Kung and Traub.

Steffensen-type methods are specially useful in real problems where the derivative can not be calculated. This is the case of nonsmooth functions; Amat and Busquier in [9] presented an strategy to control the approximation of the derivative and the stability of the iteration. They applied this idea on Steffensen's method (STM):

$$
x_{k+1}=x_{k}-\frac{f\left(x_{k}\right)}{\left[f\left(x_{k}+\alpha_{k}\left|f\left(x_{k}\right)\right| f\left(x_{k}\right)\right)-f\left(x_{k}\right)\right] / \alpha_{k}\left|f\left(x_{k}\right)\right| f\left(x_{k}\right)},
$$

where the parameters $\alpha_{k} \in \mathbb{R}$ will allow to control the approximation of the derivative. This procedure can be applied to any other derivative-free scheme. The authors showed in [9] the second-order convergence for nonsmooth functions of (10) and mentioned that, in order to control the stability in practice, the parameters $\alpha_{k}$ can be computed such that:

$$
t_{o l} \ll\left|\alpha_{k}\right| f\left(x_{k}\right)\left|f\left(x_{k}\right)\right| \leq t o l_{u},
$$

where $t o l_{c}$ is related to the computer precision and tol $l_{u}$ is a user's free parameter. This strategy is a good alternative to the proposed by Potra et al. in [10], in which the authors consider $\alpha_{k}=\epsilon, 0<\epsilon \leq 1$ fix for all $k$.

In the following section, we will apply this strategy on our proposed method, Op4, obtained a modified scheme that will be denoted as Op4mod. Then, we will analyze how its behavior improves in nonsmooth cases, although the order of convergence on singular points slows down to two. 


\section{$3 \quad$ Numerical results}

We have divided the section in two parts: firstly, we check the described methods by using smooth equations and, secondly, we analyze the performance of the methods on nonsmooth ones.

In the first part of this section, we test the effectiveness of the new optimal fourth-order family of methods (6), taking $a=b=c=1$ and $d=0$; this is compared with the classical Steffensen's method, SM, the Jain's method SSM, the method DHM presented by Dehghan and Hajarian in [3] and the optimal fourth-order method, RM, with $a=0$. These methods are employed to solve some nonlinear equations. Specifically, we use the following functions:

(a) $f(x)=\sin ^{2} x-x^{2}+1, x^{*} \approx 1.404492$,

(b) $f(x)=x^{2}-e^{x}-3 x+2, x^{*} \approx 0.257530$,

(c) $f(x)=\cos x-x, x^{*} \approx 0.739085$,

(d) $f(x)=(x-1)^{3}-1, x^{*}=2$,

(e) $f(x)=x^{3}-10, x^{*} \approx 2.154435$,

(f) $f(x)=\cos (x)-x e^{x}+x^{2}, x^{*} \approx 0.639154$,

(g) $f(x)=e^{x}-1.5-\arctan (x), x^{*} \approx 0.767653$,

(h) $f(x)=x^{3}+4 x^{2}-10, x^{*} \approx 1.365230$,

(i) $f(x)=8 x-\cos (x)-2 x^{2}, x^{*} \approx 0.128077$,

(j) $f(x)=\arctan (x), x^{*}=0$,

Numerical computations have been carried out using variable precision arithmetic, with 256 digits, in MATLAB 7.1. The stopping criterion used is $\left|x_{k+1}-x_{k}\right|+$ $\left|f\left(x_{k}\right)\right|<10^{-100}$, therefore, we check that the iterates succession converge to an approximation to the solution of the nonlinear equation. For every method, we count the number of iterations needed to reach the wished tolerance and estimate the computational order of convergence (ACOC), defined by the authors in [11]:

$$
\rho=\frac{\ln \left(\left|x_{k+1}-x_{k}\right| /\left|x_{k}-x_{k-1}\right|\right)}{\ln \left(\left|x_{k}-x_{k-1}\right| /\left|x_{k-1}-x_{k-2}\right|\right)}
$$

Table 1 shows the number of iterations required to satisfy the stopping criterion (if the method does not converge, it will be denoted by "nc") and the approximated computational order of convergence $\rho$. The value of $\rho$ that appears in this table is the last coordinate of vector $\rho$ when the variation between its values is small.

On the other hand, in Table 2 the mean elapsed time, after 1000 performances of the program, appears. 


\begin{tabular}{|c|c|c|c|c|c|c|c|c|c|c|c|}
\hline$f(x)$ & $x_{0}$ & \multicolumn{7}{|c|}{ Iterations } & \multicolumn{5}{c|}{$\rho$} \\
\hline & & SM & SSM & DHM & RM & Op4 & SM & SSM & DHM & RM & Op4 \\
\hline \hline a) & 1 & 9 & 6 & 7 & 5 & 5 & 2.00 & 3.00 & 3.00 & 4.00 & 4.00 \\
\hline b) & 0.7 & 8 & 6 & 7 & 5 & 5 & 2.00 & 3.00 & 3.00 & 4.00 & 4.00 \\
\hline c) & 1 & 8 & 5 & 6 & 5 & 5 & 2.00 & 3.00 & 3.00 & 4.00 & 4.00 \\
\hline d) & 1.5 & nc & 12 & 9 & 8 & 8 & - & 3.00 & 3.00 & 4.00 & 4.00 \\
\hline e) & 2 & 15 & 6 & 7 & 6 & 6 & 2.00 & 3.00 & 3.00 & 4.00 & 4.00 \\
\hline f) & 1 & 10 & 6 & 8 & 5 & 5 & 2.00 & 3.00 & 3.00 & 4.00 & 4.00 \\
\hline g) & 1 & 10 & 6 & 6 & 5 & 5 & 2.00 & 3.00 & 3.00 & 4.00 & 4.00 \\
\hline h) & 1.5 & 11 & 6 & 7 & 5 & 5 & 2.00 & 3.00 & 3.00 & 4.00 & 4.00 \\
\hline i) & 1 & 15 & 7 & 7 & 8 & 8 & 2.00 & 3.00 & 2.59 & 4.00 & 4.00 \\
\hline j) & 0.5 & 7 & 5 & 5 & 5 & 5 & 3.00 & 5.00 & 5.00 & 5.00 & 5.00 \\
\hline \hline Thle 1 & & & & & & & & & \\
\hline
\end{tabular}

Table 1

Numerical results for nonlinear equations from (a) to (j)

\begin{tabular}{|c|c|c|c|c|c|c|}
\hline$f(x)$ & $x_{0}$ & SM & SSM & DHM & RM & Op4 \\
\hline \hline a) & 1 & 0.1477 & 0.1523 & 0.2262 & 0.1422 & 0.1380 \\
\hline b) & 0.7 & 0.1390 & 0.1560 & 0.2335 & 0.1495 & 0.1456 \\
\hline c) & 1 & 0.0887 & 0.0883 & 0.1290 & 0.0963 & 0.0963 \\
\hline d) & 1.5 & nc & 0.2356 & 0.2201 & 0.1763 & 0.1763 \\
\hline e) & 2 & 0.1502 & 0.0958 & 0.1354 & 0.1140 & 0.1140 \\
\hline f) & 1 & 0.1764 & 0.1646 & 0.2890 & 0.1494 & 0.1494 \\
\hline g) & 1 & 0.1580 & 0.1464 & 0.1904 & 0.1341 & 0.1341 \\
\hline h) & 1.5 & 0.1758 & 0.1496 & 0.2170 & 0.1370 & 0.1370 \\
\hline i) & 1 & 0.2717 & 0.1894 & 0.2390 & 0.2355 & 0.2355 \\
\hline j) & 0.5 & 0.0791 & 0.0871 & 0.1072 & 0.0990 & 0.0990 \\
\hline \hline
\end{tabular}

Table 2

Mean e-time in 1000 performances of the program

Numerical results are in concordance with the theory developed in this paper. In both tables we can observe that the results obtained with our new method are better, not only than the obtained by the methods SM, SSM and DHM, but also improve the results from the other fourth-order optimal scheme RM, in some cases.

Now, we are going to make some numerical simulations in order to test how 
the methods SM and Op4 behave in nonsmooth cases. Moreover, we apply the $\alpha_{k}$-procedure on both methods to avoid some stability problems. In these cases, numerical computations have been carried out using simple precision arithmetic, so $t o l_{c}=10^{-16}$, and the stopping criterion used has been $\left|x_{k+1}-x_{k}\right|<t_{0} l_{u}=10^{-8}$. From a sufficiently small $\alpha_{0}$, we use the following algorithm to compute de different $\alpha_{k}$ :

$$
\alpha_{k+1}= \begin{cases}\alpha_{k}^{2}, & \text { if }\left|\alpha_{k}^{2}\right| f\left(x_{k}\right)\left|f\left(x_{k}\right)\right| \geq t_{c} l_{c} \\ \operatorname{tol}_{c} /|| f\left(x_{k}\right)\left|f\left(x_{k}\right)\right|, & \text { elsewhere. }\end{cases}
$$

The first test has been made on the function:

$$
f(x)= \begin{cases}x(x+1), & \text { if } x<0, \\ -2 x(x-1), & \text { if } x \geq 0\end{cases}
$$

that can be found in [12]. We use three initial estimations in order to approximate the three different roots of the equation $\{0,1,-1\}$. In Table 3 we show for each initial estimations and every method, the exact absolute error at first and last iterations, the absolute difference between the two last iterations (denoted by incr) and the ACOC. Let us note that when the approximated convergence order is not stable, we will denote it by '-'. From Table 3 can be inferred that the order of convergence of Op4 method slows down to two when it is applied on nonsmooth equations. Nevertheless, it usually performs better or equal than Steffensen's method and its modifications by the $\alpha_{k}$ procedure. Indeed, when this strategy is applied on the fourth-order method (Op4mod), the stability of the method is improved and it results in more precise estimations with lower number of iterations. Moreover, the ACOC is also stabilized in $\rho \approx 4$, except in the singular case: around $x^{*}=0$, the convergence is still quadratic.

Let us now make a chance for the following nonsmooth function, that can be found in $[9]$ :

$$
f(x)= \begin{cases}10\left(x^{4}+x\right), & \text { if } x<0, \\ -10\left(x^{3}+x\right), & \text { if } x \geq 0 .\end{cases}
$$

The numerical experiments made on (13) are summarized in Table 4. In this case, the advantages of the modified methods over original Steffensen's method are more evident when the initial estimation is far from the zero of the function. As $x^{*}=0$ is a multiple root of (13), the estimated order of convergence is around 3, when is stable. When the initial estimation is good enough, it is clear that the behavior of Op4 and Op4mod improves lower-order methods, in terms of precision and number of iterations. 


\begin{tabular}{|c|c|c|c|c|c|c|c|c|c|c|}
\hline & \multicolumn{2}{|l|}{$\mathrm{SM}$} & \multicolumn{2}{|c|}{$\epsilon=10^{-8}$} & \multicolumn{2}{|c|}{$\operatorname{STM}\left(t_{0} l_{u}=10^{-8}\right)$} & \multicolumn{2}{|c|}{ Op4 } & \multicolumn{2}{|c|}{ Op4mod } \\
\hline$x_{0}=0.1$ & iter & error & iter & error & iter & error & iter & error & iter & error \\
\hline \multirow{5}{*}{$x^{*}=0$} & 1 & $4.52 \mathrm{e}-2$ & 1 & $1.25 \mathrm{e}-2$ & 1 & $1.25 \mathrm{e}-2$ & 1 & $1.93 \mathrm{e}-2$ & 1 & $5.74 \mathrm{e}-3$ \\
\hline & 2 & $4.60 \mathrm{e}-3$ & 2 & $1.60 \mathrm{e}-4$ & 2 & $1.60 \mathrm{e}-4$ & 2 & $6.95 \mathrm{e}-4$ & 2 & $3.25 \mathrm{e}-5$ \\
\hline & $\vdots$ & & $\vdots$ & & $\vdots$ & & $\vdots$ & & 3 & $1.06 \mathrm{e}-9$ \\
\hline & 4 & $8.32 \mathrm{e}-9$ & 4 & 0 & 4 & 0 & 4 & $1.86 \mathrm{e}-12$ & 4 & 0 \\
\hline & 5 & 0 & 5 & $\mathrm{NaN}$ & 5 & 0 & 5 & 0 & & \\
\hline incr & \multicolumn{2}{|c|}{$8.32 \mathrm{e}-9$} & \multicolumn{2}{|c|}{$\mathrm{NaN}$} & \multicolumn{2}{|c|}{$1.10 \mathrm{e}-15$} & \multicolumn{2}{|c|}{$1.86 \mathrm{e}-12$} & \multicolumn{2}{|c|}{$1.06 \mathrm{e}-9$} \\
\hline$\rho$ & \multicolumn{2}{|c|}{2.0919} & \multicolumn{2}{|l|}{ - } & \multicolumn{2}{|c|}{1.9756} & \multicolumn{2}{|c|}{2.0000} & \multicolumn{2}{|c|}{1.9998} \\
\hline$x_{0}=3$ & iter & error & iter & error & iter & error & iter & error & iter & error \\
\hline \multirow{5}{*}{$x^{*}=1$} & 1 & 0.29 & 1 & 0.80 & 1 & 0.80 & 1 & 0.18 & 1 & 0.25 \\
\hline & 2 & 0.15 & 2 & 0.25 & 2 & 0.25 & 2 & $2.13 \mathrm{e}-3$ & 2 & $1.52 \mathrm{e}-3$ \\
\hline & $\vdots$ & & $\vdots$ & & $\vdots$ & & 3 & $2.09 \mathrm{e}-11$ & 3 & $5.37 \mathrm{e}-12$ \\
\hline & 6 & $1.00 \mathrm{e}-14$ & 5 & $2.32 \mathrm{e}-6$ & 6 & $5.35 \mathrm{e}-12$ & 4 & 0 & 4 & 0 \\
\hline & 7 & 0 & 6 & $\mathrm{NaN}$ & 7 & 0 & & & & \\
\hline incr & \multicolumn{2}{|c|}{$6.33 \mathrm{e}-15$} & \multicolumn{2}{|c|}{$\mathrm{NaN}$} & \multicolumn{2}{|c|}{$5.35 \mathrm{e}-12$} & \multicolumn{2}{|c|}{$2.09 \mathrm{e}-11$} & \multicolumn{2}{|c|}{$5.37 \mathrm{e}-12$} \\
\hline$\rho$ & \multicolumn{2}{|c|}{2.0008} & \multicolumn{2}{|l|}{ - } & \multicolumn{2}{|c|}{2.0007} & \multicolumn{2}{|l|}{-} & \multicolumn{2}{|c|}{3.8331} \\
\hline$x_{0}=-10$ & iter & error & iter & error & iter & error & iter & error & iter & error \\
\hline \multirow{5}{*}{$x^{*}=-1$} & 1 & 8.36 & 1 & 4.26 & 1 & 4.26 & 1 & 4.29 & 1 & 1.91 \\
\hline & 2 & 7.71 & 2 & 1.91 & 2 & 1.91 & 2 & 1.82 & 2 & 0.23 \\
\hline & $\vdots$ & & $\vdots$ & & $\vdots$ & & $\vdots$ & & $\vdots$ & \\
\hline & 13 & 0 & 7 & $1.38 \mathrm{e}-6$ & 8 & $1.92 \mathrm{e}-12$ & 5 & $1.08 \mathrm{e}-9$ & 4 & $1.93 \mathrm{e}-12$ \\
\hline & 14 & 0 & 8 & $\mathrm{NaN}$ & 9 & 0 & 6 & $\mathrm{NaN}$ & 5 & 0 \\
\hline incr & \multicolumn{2}{|c|}{$4.66 \mathrm{e}-15$} & \multicolumn{2}{|c|}{$\mathrm{NaN}$} & \multicolumn{2}{|c|}{$1.92 \mathrm{e}-12$} & $\mathrm{NaN}$ & & 1.93 & -12 \\
\hline$\rho$ & - & & - & & 2.00 & & - & & 3.84 & \\
\hline
\end{tabular}

Table 3

Numerical results for function (12)

\section{Conclusions}

We have obtained a new one-parameter fourth-order family of iterative methods for solving nonlinear equations. Each member of this class requires only three functional evaluations per step, so its efficiency index is equal to $4^{1 / 3}=$ 


\begin{tabular}{|c|c|c|c|c|c|c|c|c|c|c|}
\hline \multirow{2}{*}{$x_{0}=32$} & \multicolumn{2}{|l|}{$\mathrm{SM}$} & \multicolumn{2}{|c|}{$\epsilon=10^{-8}$} & \multicolumn{2}{|c|}{$\operatorname{STM}\left(\operatorname{tol}_{u}=10^{-8}\right)$} & \multicolumn{2}{|c|}{ Op4 } & \multicolumn{2}{|c|}{ Op4mod } \\
\hline & iter & error & iter & error & iter & error & iter & error & iter & error \\
\hline \multirow{5}{*}{$x^{*}=0$} & \multirow{5}{*}{\multicolumn{2}{|c|}{$>10^{4}$}} & 1 & 31.97 & 1 & 21.33 & 1 & 22.0 & 1 & 13.56 \\
\hline & & & 2 & 31.95 & 2 & 14.21 & 2 & 14.56 & 2 & 5.71 \\
\hline & & & $\vdots$ & & $\vdots$ & & $\vdots$ & & $\vdots$ & \\
\hline & & & 149 & $1.59 \mathrm{e}-10$ & 13 & 0 & 10 & $2.03 \mathrm{e}-12$ & 6 & $4.23 \mathrm{e}-11$ \\
\hline & & & 150 & $4.0 \mathrm{e}-11$ & 14 & 0 & 11 & 0 & 7 & 0 \\
\hline incr & & & \multicolumn{2}{|c|}{$1.99 \mathrm{e}-10$} & \multicolumn{2}{|c|}{$1.68 \mathrm{e}-18$} & \multicolumn{2}{|c|}{$2.03 \mathrm{e}-12$} & \multicolumn{2}{|c|}{$4.23 \mathrm{e}-11$} \\
\hline$\rho$ & & & \multicolumn{2}{|l|}{-} & \multicolumn{2}{|c|}{2.9985} & \multicolumn{2}{|l|}{-} & \multicolumn{2}{|l|}{-} \\
\hline$x_{0}=16$ & iter & error & iter & error & iter & error & iter & error & iter & error \\
\hline \multirow{5}{*}{$x^{*}=0$} & \multirow{5}{*}{\multicolumn{2}{|c|}{$>10^{4}$}} & 1 & 13.80 & 1 & 10.65 & 1 & 10.65 & 1 & 6.75 \\
\hline & & & 2 & 10.89 & 2 & 7.08 & 2 & 7.08 & 2 & 2.79 \\
\hline & & & $\vdots$ & & $\vdots$ & & $\vdots$ & & $\vdots$ & \\
\hline & & & 12 & 1.0 e-10 & 11 & $1.2 \mathrm{e}-13$ & 10 & $4.0 \mathrm{e}-14$ & 6 & 0 \\
\hline & & & 13 & $3.75 \mathrm{e}-11$ & 12 & 0 & 11 & 0 & 7 & 0 \\
\hline incr & & & \multicolumn{2}{|c|}{$6.26 \mathrm{e}-11$} & \multicolumn{2}{|c|}{$1.16 \mathrm{e}-13$} & \multicolumn{2}{|c|}{$4.35 \mathrm{e}-14$} & \multicolumn{2}{|c|}{$7.85 \mathrm{e}-32$} \\
\hline$\rho$ & & & \multicolumn{2}{|c|}{3.2225} & \multicolumn{2}{|c|}{3.0003} & \multicolumn{2}{|l|}{-} & \multicolumn{2}{|l|}{-} \\
\hline$x_{0}=1$ & iter & error & iter & error & iter & error & iter & error & iter & error \\
\hline \multirow{5}{*}{$x^{*}=0$} & 1 & 1.0 & 1 & 0.5 & 1 & 0.5 & 1 & 0.49 & 1 & $8.33 \mathrm{e}-2$ \\
\hline & 2 & 0.99 & 2 & 0.14 & 2 & 0.14 & 2 & 0.11 & 2 & $7.71 \mathrm{e}-6$ \\
\hline & $\vdots$ & & $\vdots$ & & $\vdots$ & & $\vdots$ & & & 0 \\
\hline & 98 & $3.0 \mathrm{e}-13$ & 5 & $3.19 \mathrm{e}-12$ & 5 & 0 & 4 & $2.3 \mathrm{e}-13$ & 4 & 0 \\
\hline & 99 & 0 & 6 & $\mathrm{NaN}$ & 6 & 0 & 5 & 0 & & \\
\hline incr & \multicolumn{2}{|c|}{$3.03 \mathrm{e}-13$} & \multicolumn{2}{|c|}{$\mathrm{NaN}$} & \multicolumn{2}{|c|}{$7.66 \mathrm{e}-20$} & \multicolumn{2}{|c|}{$2.34 \mathrm{e}-13$} & $7.87 €$ & -32 \\
\hline$\rho$ & - & & - & & 2.99 & & - & & - & \\
\hline
\end{tabular}

Table 4

Numerical results for function (13)

1.587. Therefore, the family of methods agrees with the conjecture of KungTraub for $d=3$. In addition, these methods are derivative-free, which allow us to apply them also on nonsmooth equations with positive and promising results. The generalization of these methods to nonlinear systmes $F(x)=0$ is 
similar to the classical Steffensen's method (see [1]:

$$
\begin{aligned}
& \tilde{x}_{k}=x_{k}+\alpha_{k}\left\|F\left(x_{k}\right)\right\| F\left(x_{k}\right), \\
& x_{k+1}=x_{k}-\left[x_{k}, \tilde{x}_{k} ; F\right]^{-1} F\left(x_{k}\right)
\end{aligned}
$$

where $[u, v ; F]: \mathbb{R}^{n} \rightarrow \mathbb{R}^{n}$ is a linear operator such that $[u, v ; F](u-v)=$ $F(u)-F(v)$ is called divided difference.

\section{Acknowledgement}

The authors would like to thank the referee for the valuable comments and for the suggestions to improve the readability of the paper.

\section{References}

[1] J.M. Ortega, W.G. Rheinboldt, Iterative solutions of nonlinear equations in several variables, Academic Press, New York, 1970.

[2] P. Jain, Steffensen type methods for solving nonlinear equations, Applied Mathematics and Computation, 194 (2007) 527-533.

[3] M. Dehghan, M. Hajarian, An Some derivative free quadratic and cubic convergence iterative formulas for solving nonlinear equations, Journal of Computational and Applied Mathematics, 29 (2010) 19-30.

[4] H. Ren, Q. Wu, W. Bi, A class of two-step Steffensen type methods with fourthorder convergence, Applied Mathematics and Computation, 209 (2009) 206-210.

[5] Q. Zheng, J. Wang, P. Zhao, L. Zhang, A Steffensen-like method and its higherorder variants, Applied Mathematics and Computation, 214 (2009) 10-16.

[6] X. Feng, Y. He, High order oterative methods without derivatives for solving nonlinear equations, Applied Mathematics and Computation, 186 (2007) 16171623 .

[7] A.M. Ostrowski, Solutions of equations and systems of equations, Academic Press, New York-London, 1966.

[8] H.T. Kung, J.F. Traub, Optimal order of one-point and multi-point iteration, Applied Mathematics and Computation, 21 (1974) 643-651.

[9] S. Amat, S. Busquier, On a Steffensen's type method and its behavior for semismooth equations, Applied Mathematics and Computation, 177 (2006) 819823.

[10] F.A. Potra, L. Qi, D. Sun Secant methods for semismooth equations, Numer. Math., 80 (1998) 305-324. 
[11] A. Cordero, J.R. Torregrosa, Variants of Newton's method using fifth-order quadrature formulas, Applied Mathematics and Computation, 190 (2007) 686698.

[12] S. Amat, S. Busquier, On a higher order secant methods, Applied Mathematics and Computation, 141 (2003) 321-329. 